\title{
Transcriptomic comparative analysis of hippocampal tissue and primary cultures after hyaluronidase treatment
}

\author{
V. Pershin ${ }^{1,2 *}$, A. Balashova ${ }^{2}$, E. Guryev ${ }^{1,2}$, L. Kurbatov ${ }^{3}$, M. Gainullin ${ }^{1,4}$, \\ I. Mukhina ${ }^{1,2}$ \\ ${ }^{1}$ Privolzhsky Research Medical University, Nizhny Novgorod, Russia \\ ${ }^{2}$ Lobachevsky State University, Nizhny Novgorod, Russia \\ ${ }^{3}$ Orekhovich Institute of Biomedical chemistry, Russian Academy of Medical Sciences, Moscow, Russia \\ ${ }^{4}$ Oslo University Hospital, Oslo, Norway \\ *e-mail:bp1995@yandex.ru
}

Key words: Extracellular matrix, epilepsy, hippocampus, gene expression, transcriptomics.

Motivation and Aim: It is well-known that traumatic injury or enzymatic digestion of brain extracellular matrix can cause seizure-like activity in primary hippocampal culture [1]. However, underlying molecular mechanism and similarity between in vitro and in vivo models of epilepsy is poorly investigated. The aim of this study was to investigate changes of gene expression and perform comparative transcriptomic analysis while modeling of epileptogenesis.

Methods and Algorithms: C57BL/6J mice were used to prepare hippocampal cell culture and intra-hippocampal hyaluronidase injection. Hyaluronic acid, which is the basis of extracellular matrix, has been removed by $75 \mathrm{U} / \mathrm{ml}$ hyaluronidase. Transcriptomic analysis was performed by mouse full-genome 2-colour Agilent Microarrays. Normalization, processing and detection of differentially-expressed genes were performed by online free software - Babelomics 5.0 [2]. Functional annotation clustering was made by David bioinformatics resource [3].

Results: Hyaluronan digestion resulted in significant changes of expression of 3 gene clusters in neuronal hippocampal cultures - synaptic gene cluster, ribosomal gene cluster and mitochondrial gene cluster, while in hippocampus hyaluronidase injection resulted in changes of such functional gene clusters: synaptic gene cluster, neurogenesis gene cluster, actin-binding gene cluster, circadian rhythm cluster and ubiquitinylation gene cluster. When comparing control groups of in vivo and in vitro samples there were found 3 significant gene clusters: calcium ion-binding cluster, cluster of genes expressing EGFdomains and cortical cytoskeleton gene cluster.

Conclusion: The results obtained in this study suggest that hyaluronic acid digestion mainly affect synaptic gene cluster that indicate to similarity of in vivo and in vitro models. A wide variety of gene clusters from isolated hippocampus point to high complexity of biological processes in mice brain. Transcriptomic comparative analysis revealed strong difference between cell culture and hippocampus that should be considered when interpreting in vitro data.

Acknowledgements: Supported by the RFBR (16-04-01763).

\section{References}

1. Vedunova M.V. et al. (2013) Seizure-like activity in hyaluronidase-treated dissociated hippocampal cultures. Frontiers in cellular Neuroscience. 7(149):81-89. DOI 10.3389/fncel.2013.00149.

2. Alonso R. et al. (2015) Babelomics 5.0: functional interpretation for new generations of genomic data. Nucleic Acids Res. 43(1):117-121.

3. Huang D.W. et al. (2009) Systematic and integrative analysis of large gene lists using DAVID Bioinformatics Resources. Nature Protoc. 4(1):44-57. 